\title{
How much should I eat? A comparison of suggested portion sizes in the UK
}

\author{
Hannah B Lewis*, Amy L Ahern and Susan A Jebb \\ MRC Human Nutrition Research, Elsie Widdowson Laboratory, Fulbourn Road, Cambridge CB1 9NL, UK
}

Submitted 14 October 2011: Final revision received 29 February 2012: Accepted 6 March 2012: First published online 27 April 2012

\begin{abstract}
Objective: To identify and compare suggested food portion sizes in UK schemes. Design: The study collated and compared suggested portion sizes from selected UK schemes intended both for general advice and weight-loss advice.

Setting: Portion size schemes were included if they were relevant to the UK, provided actual portion size information, were intended for adults and were obtainable from the public domain in November 2010. Included schemes were from the food industry, non-governmental organisations and health-care professionals. Suggested portion sizes of foods occurring in at least one scheme for general advice and at least one scheme for weight loss were included. Own brand on-pack portion size labelling from a large UK-wide supermarket was added to represent portion size advice from UK food retailers.

Subjects: Not applicable.

Results: The suggested portion sizes in the weight-loss advice schemes were often concordant, as were the general advice schemes, except one general advice scheme from a non-governmental organisation which was more closely aligned with the portion sizes for weight loss. Overall there were substantial discrepancies between suggested portion sizes for muesli and crunchy breakfast cereals, rice, pasta and potatoes, meat, fish and pulses, whereas portion sizes for cooked vegetables, dried fruit, some breakfast cereals and cheese were broadly consistent. Conclusions: There is a lack of consistency in the portion sizes communicated to the public. An independent and authoritative scheme of suggested portion sizes for all foods, with distinct recommendations for general advice and for weight-loss advice, could be of benefit.
\end{abstract}

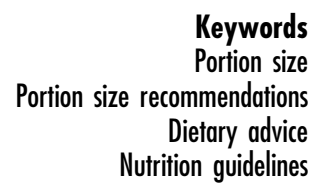

Portion size is defined as the amount of food an individual intends to consume at one eating occasion ${ }^{(1)}$. It is an important determinant of energy intake ${ }^{(2)}$ and larger than appropriate portion sizes could increase the risk of weight gain ${ }^{(3)}$. Secular trends that favour greater availability of larger portions may be contributing to the concept of 'portion distortion', whereby larger portions are perceived as appropriate to consume ${ }^{(4)}$.

Conveying information on appropriate portion size could help facilitate appropriate intakes. Schemes for the communication of portion size messages have been described in a report by the Institute of Grocery Distribution (IGD) ${ }^{(1)}$. Portion size schemes were reported to have been developed for several purposes: analysing dietary intakes, production of menus, communication to consumers, legislation, weight loss or as an aid to achieving a healthy $\operatorname{diet}^{(1)}$. However it was reported that the public is reluctant to give much heed to portion size information on labels ${ }^{(5)}$. Other research also reported concerns about the low credibility of portion size information to consumers ${ }^{(6)}$.
A lack of clear communication of recommended portion sizes could contribute towards the inability to form appropriate concepts of suitable portion sizes. Moreover, inconsistent portion sizes could generate doubts over the validity of portion size schemes. One possible reason why portion size information may not be credible could be a lack of consistency across suggested portion sizes. Therefore, it is important to examine the consistency of suggested portion sizes communicated to the public to clarify whether there is scope for improvement in the communication of food portion sizes. The current study identified and compared suggested portion sizes from selected schemes, both for general advice and for weight-loss advice.

\section{Method}

\section{Study design}

Suggested portion sizes for UK schemes were collated and compared. Due to the nature of the data, statistical analysis was not appropriate.

\footnotetext{
*Corresponding author: Email Hannah.Lewis@mrc-hnr.cam.ac.uk
}

(C) The Authors 2012. The online version of this article is published within an Open Access environment subject to the conditions of the Creative Commons Attribution-NonCommercial-ShareAlike licence $<$ http://creativecommons.org/licenses/by-nc-sa/3.0/ $>$. The written permission of Cambridge University Press must be obtained for commercial re-use. 


\section{Scheme selection}

Portion size schemes were identified from the IGD report ${ }^{(1)}$ and selected for inclusion if they were named schemes in, or relevant to, the UK that provided actual portion size information and were obtainable from the public domain in November 2010. Schemes were excluded if they were aimed at children, people in hospital or the elderly; if they stated frequency of portions but not portion size; and if they included suggestions only for a single food group (e.g. the Dairy Council or the National Health Service (NHS) 5-a-day scheme). As the IGD report included unnamed retailers and businesses, it was not possible to identify the source of these schemes. Therefore three large UK-wide supermarkets, as examples of retailers, were approached independently and asked to contribute their portion size information; only one supermarket provided the required information. Own brand on-pack portion size labelling on the nutrition information panel provided by this UK supermarket was used to represent portion size advice from UK food retailers. The Confederation of the Food and Drink Industries of the EU (CIAA) ${ }^{(7)}$ scheme included opinions of the European Union-wide regulatory bodies for the manufacture of several food types ${ }^{(8-16)}$. As many schemes mentioned in the IGD report were not obtainable or applicable, the British Dietetic Association (BDA) and British Heart Foundation (BHF) schemes were included to improve the breadth of the review. These are two trusted UK organisations from which the public receives portion size guidance often via literature provided by health professionals. Selected schemes are shown in Table 1 and were divided into two purposes (general advice and weight-loss advice) and three categories, pertaining to the origins of the scheme (industry, health-care professionals (HCP) and non-governmental organisations (NGO)).

\section{Portion size calculations}

Available portion sizes were collated and, where appropriate, allocated to a generic rather than a specific food category (e.g. carrots were allocated to the 'vegetables' category). Foods were included when they occurred in at least one scheme for each scheme purpose (i.e. in one for general advice and one for weight loss) considering all schemes except the retailer scheme. The retailer portion sizes were added for each food already chosen for analysis. For each food, the standard products were identified for inclusion (e.g. for the bread category, gluten-free bread, part-baked bread, rolls, speciality breads and fruit breads were not included) and the median and range for the portion sizes were taken, as most data did not appear to be normally distributed.

Where portion sizes were optional depending on the energy (calorie) level desired, such as those for the snacks in the BDA scheme, these were excluded. Household measures such as tablespoons were converted to gram weights by an independent observer using conversions from the dietary assessment system DINO (Diet In Nutrients Out) developed at MRC Human Nutrition Research, based on Food Standards Agency portion sizes book ${ }^{(17)}$ and direct observation as required. Where a suggested portion size was given for a general food without specific examples, a range of conversions was used and the median was taken (e.g. for the suggested portion size ' 1 piece of medium fruit', the median of apple, pear, orange, peach and banana weights was taken). Portion sizes that were given as weight of a raw food, when the food would be cooked before consuming, were converted into portion sizes of the cooked product ${ }^{(18)}$.

There was general inconsistency as to use of the terms 'serving' and 'portion' sizes in the starchy carbohydratebased foods group. Often, portion sizes were specified as a serving size and a frequency. As such, the scheme intends the number of eating occasions and the serving size to be considered when following the advice. For example, the Diabetes UK scheme recommends that women aiming to achieve an energy intake of $6276 \mathrm{~kJ} / \mathrm{d}(1500 \mathrm{kcal} / \mathrm{d})$ should consume five servings of foods from this category daily. Thus, unless five separate meals are consumed where each time starchy carbohydrates are included, the total portion size must be more than one serving. Therefore, for schemes where two servings of starchy carbohydrates are intended to be consumed at one eating occasion (World Cancer Research Fund (WCRF), Diabetes UK, BHF, BDA, Dietitians in Obesity Management UK (DOM UK)), the serving size specified was doubled to represent the realistic portion size.

\section{Results}

The suggested portion sizes are shown in Table 2. For many food types a single suggested portion size was given by many schemes. Where a suggested portion size was given as a range, the midpoint and range are shown. The retailer portion sizes are reported as a median and range unless all relevant products for a food gave the same suggested portion size.

\section{Fruit and vegetables}

For the fruit and vegetable food group, the portion sizes were very consistent across NGO schemes, which, in general, were also consistent across all the HCP schemes. However, the portion sizes were often larger than the $80 \mathrm{~g}$ recommendation from the NHS 5-a-day scheme. Compared with the NGO schemes, the retailer portion sizes were 55\% smaller for salad vegetables and 30\% smaller for medium fruit. Retailer portion sizes were consistent for cooked vegetables (90 to $100 \mathrm{~g}$ ), berries and grapes (72 to $80 \mathrm{~g}$ ) and dried fruit (30 g), but on average $60 \%$ larger for fruit juice and $60 \%$ larger, and with more variability $(80-400 \mathrm{~g}$ ), for large fruit. The retailer portion size for large fruit was also considerably larger when comparing it with the retailer portion sizes for berries and 
Table 1 Suggested portion size schemes used, in the relevant purpose types and categories, with the target population and basis of the portion sizes in the scheme

\begin{tabular}{|c|c|c|c|c|c|}
\hline Scheme type & Scheme category & Author & Scheme title & Target population & Basis of scheme \\
\hline \multirow[t]{3}{*}{ General advice } & Industry & UK food retailer & $\begin{array}{l}\text { The retailer's own product, on-pack } \\
\text { serving sizes and nutritional } \\
\text { information* }\end{array}$ & Consumers & $\begin{array}{l}\text { - Pack size } \\
\text { - 'What is reasonable' } \\
\text { - FSA Food Portion Sizes book } \\
\text { - Per item } \\
\text { - From industry guidancet }\end{array}$ \\
\hline & Industry & CIAA & $\begin{array}{l}\text { 'Members portion size rationale for } \\
\text { the Guideline Daily Amounts (GDA) } \\
\text { labelling scheme'(7) }\end{array}$ & EU consumers & 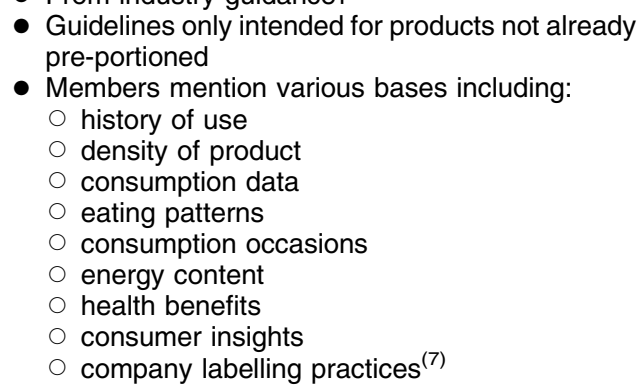 \\
\hline & NGO & WCRF & $\begin{array}{l}\text { Portion Pad web article 'What does a } \\
\text { healthy portion look like?'(27) }\end{array}$ & All adults & Not available \\
\hline \multirow[t]{4}{*}{ Weight-loss advice } & NGO & Diabetes UK & 'Calorie controlled plan' web page ${ }^{(28)}$ & $\begin{array}{l}\text { Adults (with diabetes) } \\
\text { who want to lose } \\
\text { weight }\end{array}$ & $\begin{array}{l}\text { Per portion: } \\
\text { - } 188 \mathrm{~kJ}(45 \mathrm{kcal}) \text { for fruit and vegetables } \\
\text { - } 377 \mathrm{~kJ}(90 \mathrm{kcal}) \text { for starchy carbohydrates } \\
\text { - } 314 \mathrm{~kJ}(75 \mathrm{kcal}) \text { for dairy } \\
\text { - } 586 \mathrm{~kJ}(140 \mathrm{kcal}) \text { for meats and alternatives } \\
\text { - } 356 \mathrm{~kJ}(85 \mathrm{kcal}) \text { for fats and sugars }{ }^{(28)}\end{array}$ \\
\hline & NGO & $\mathrm{BHF}$ & $\begin{array}{l}\text { 'So you want to lose weight... for } \\
\text { good' publication }{ }^{(20)}\end{array}$ & $\begin{array}{l}\text { Adults wanting to lose } \\
\text { weight }\end{array}$ & $\begin{array}{l}\text { Per portion (according to the EatWell plate food } \\
\text { group contributions): } \\
\text { - } 167 \mathrm{~kJ}(40 \mathrm{kcal}) \text { for fruit and vegetables } \\
\text { - } 335 \mathrm{~kJ}(80 \mathrm{kcal}) \text { for starchy carbohydrates } \\
\text { - } 377 \mathrm{~kJ}(90 \mathrm{kcal}) \text { for dairy } \\
\text { - } 586 \mathrm{~kJ}(140 \mathrm{kcal}) \text { for meats and alternatives } \\
\text { - } 167 \mathrm{~kJ}(40 \mathrm{kcal}) \text { for foods HFSS } \ddagger\end{array}$ \\
\hline & $\mathrm{HCP}$ & BDA & $\begin{array}{l}\text { Weight Wise - 'Eating well: your } \\
\text { weight wise plan' web page }{ }^{(21)}\end{array}$ & $\begin{array}{l}\text { Adults wanting to lose } \\
\text { weight }\end{array}$ & Not available \\
\hline & $\mathrm{HCP}$ & DOM UK & $\begin{array}{l}\text { 'Controlling your portions' - DOM UK } \\
\text { Weight Management Diet sheet }\end{array}$ & $\begin{array}{l}\text { Adults wanting to lose } \\
\text { weight }\end{array}$ & $\begin{array}{l}\text { Per portion: } \\
\text { - } 167 \mathrm{~kJ}(40 \mathrm{kcal}) \text { for fruit and vegetables } \\
\text { - } 335 \mathrm{~kJ}(80 \mathrm{kcal}) \text { for starchy carbohydrates } \\
\text { - } 377 \mathrm{~kJ}(90 \mathrm{kcal}) \text { for dairy } \\
\text { - } 586 \mathrm{~kJ}(140 \mathrm{kcal}) \text { for meat and alternatives } \\
\text { - } 209 \mathrm{~kJ}(50 \mathrm{kcal}) \text { for foods HFSS }{ }^{(22)}\end{array}$ \\
\hline
\end{tabular}

†Personal comn
are based. 
Table 2 Weight $(\mathrm{g})$ of suggested portion sizes from UK schemes

\begin{tabular}{|c|c|c|c|c|c|c|c|c|c|c|c|c|c|}
\hline \multirow[b]{4}{*}{ Food category/food } & \multicolumn{6}{|c|}{ General advice portion sizes (g) } & \multicolumn{7}{|c|}{ Weight-loss advice portion sizes (g) } \\
\hline & \multicolumn{4}{|c|}{ Industry } & \multicolumn{6}{|c|}{ NGO } & \multicolumn{3}{|c|}{$\mathrm{HCP}$} \\
\hline & \multicolumn{2}{|c|}{ Retailer } & \multicolumn{2}{|c|}{ CIAA } & \multicolumn{2}{|c|}{ WCRF } & \multicolumn{2}{|c|}{ Diabetes UK } & \multicolumn{2}{|c|}{$\mathrm{BHF}$} & \multicolumn{2}{|c|}{ BDA } & \multirow{2}{*}{$\begin{array}{c}\text { DOM UK } \\
\text { Median o } \\
\text { midpoint }\end{array}$} \\
\hline & $\begin{array}{l}\text { Median or } \\
\text { midpoint }\end{array}$ & Range & $\begin{array}{l}\text { Median or } \\
\text { midpoint }\end{array}$ & Range & $\begin{array}{l}\text { Median or } \\
\text { midpoint }\end{array}$ & Range & $\begin{array}{l}\text { Median or } \\
\text { midpoint }\end{array}$ & Range & $\begin{array}{l}\text { Median or } \\
\text { midpoint }\end{array}$ & Range & $\begin{array}{l}\text { Median or } \\
\text { midpoint }\end{array}$ & Range & \\
\hline \multicolumn{14}{|l|}{ Fruit and vegetables } \\
\hline Vegetables & 100 & $50-250$ & & & $90^{*}$ & & $90^{*}$ & & $90^{*}$ & & $90^{*}$ & & $90^{*}$ \\
\hline Salad vegetables & 42 & $30-125$ & & & $98^{*}$ & & $98^{*}$ & & $98^{*}$ & & $85^{*}$ & & $85^{\star}$ \\
\hline Fruit - berries/grapes & 80 & $80-100$ & & & $72^{*}$ & & $72^{*}$ & & $72^{*}$ & & $103^{*}$ & & $65^{*}$ \\
\hline Fruit - small & & & & & $110^{*}$ & & $110^{*}$ & & $110^{*}$ & & $138^{*}$ & $110-165$ & $153^{*}$ \\
\hline Fruit - medium & 80 & $80-85$ & & & $110^{*}$ & & $110^{*}$ & & $110^{*}$ & & $110^{*}$ & & $110^{*}$ \\
\hline Fruit - large & 200 & $80-400$ & & & $125^{*}$ & & $125^{*}$ & & $125^{*}$ & & $100^{*}$ & & $40^{*}$ \\
\hline Fruit - dried & 30 & $14-50$ & & & $30^{\star}$ & & & & $30^{*}$ & & $30^{*}$ & & $26^{*}$ \\
\hline Fruit juice & 250 & $200-330$ & & & & & 156 & & 156 & & 156 & & 156 \\
\hline \multicolumn{14}{|l|}{ Starchy carbohydrates } \\
\hline Cereal - muesli & 50 & & 45 & & $105^{\star} \dagger$ & $90-120$ & $90^{*}+$ & $60-120$ & $60^{*} \dagger$ & & $90^{*}+$ & & $90^{*}+$ \\
\hline Cereal - flakes & 30 & $30-45$ & 35 & $30-40$ & $42^{*} \dagger$ & $36-48$ & $36^{*}+$ & $24-48$ & $36^{*} \dagger$ & & $36^{*} \dagger$ & & $36^{\star}+$ \\
\hline Cereal - crunchy & 50 & & 45 & & $70^{*}+$ & $60-80$ & $60^{*}+$ & $40-80$ & & & $60^{*}+$ & & $60^{*}+$ \\
\hline Cereal - puffed rice & 30 & & 35 & $30-40$ & $28^{*}+$ & $24-32$ & $24^{\star}+$ & $16-32$ & $24^{*}+$ & & $24^{*} \dagger$ & & $24^{*}+$ \\
\hline Cereal - biscuits & 38 & $30-38$ & $42^{*}$ & & & & & & $42^{*}+$ & & & & \\
\hline Bread & 44 & $23-145$ & & & $80+$ & & $72^{\star} \dagger$ & & $72^{*}+$ & & $72^{\star}+$ & & $72^{*} \dagger$ \\
\hline Rice & 208 & $125-208$ & & & $200^{*}+$ & $160-240$ & $160^{\star} \dagger$ & & $160^{*}+$ & & $160^{*}+$ & & $160^{*} \dagger$ \\
\hline Pasta & 167 & $114-380$ & 200 & $180-220$ & $185^{\star}+$ & $148-222$ & $148^{*}+$ & & $222^{*} \dagger$ & & $222^{*}+$ & & $148^{*} \dagger$ \\
\hline Potatoes & 125 & $50-250$ & & & $240^{*}+$ & & $160^{*}+$ & & $160^{*}+$ & & $160^{*}+$ & & $160^{*} \dagger$ \\
\hline \multicolumn{14}{|c|}{ Meat, fish, legumes, nuts and seeds } \\
\hline Meat & 47 & $8-200$ & 100 & & 80 & & 73 & $60-85$ & 75 & $60-90$ & 75 & $60-90$ & 50 \\
\hline Poultry & 100 & $8-210$ & 100 & & 80 & & 73 & $60-85$ & 75 & $60-90$ & 75 & $60-90$ & 50 \\
\hline Fish - oily & 75 & $16-171$ & & & 80 & & 130 & $120-140$ & 140 & & 75 & $60-90$ & 50 \\
\hline Fish - white & 124 & $101-157$ & & & 150 & & 130 & $120-140$ & 150 & & 150 & & 125 \\
\hline Pulses & 128 & $80-210$ & & & $150^{*}$ & & $53^{*}$ & & $120^{\star}$ & & $120^{*}$ & & $150^{\star}$ \\
\hline Nuts & 25 & $25-30$ & 30 & & $50^{\star}$ & & $50^{\star}$ & & $50^{*}$ & & & & $40^{*}$ \\
\hline Seeds & 25 & $25-30$ & & & $24^{*}$ & & & & & & & & $40^{\star}$ \\
\hline \multicolumn{14}{|l|}{ Dairy } \\
\hline Milk & 200 & & & & 259 & $207-310$ & 197 & & 207 & & 207 & & 207 \\
\hline Yoghurt/fromage frais & 150 & $100-150$ & & & 162 & & 135 & & 150 & & $100^{*}$ & & 125 \\
\hline Cheese & 30 & 13-33 & & & 30 & & & & 30 & & 30 & & 25 \\
\hline \multicolumn{14}{|l|}{ Fats } \\
\hline Oil & 15 & & & & $3^{*}$ & & $6^{*}$ & & $3^{*}$ & & $3^{*}$ & & $3^{*}$ \\
\hline Butter & 10 & & & & $5^{*}$ & & $10^{*}$ & & $5^{*}$ & & $5^{\star}$ & & $5^{*}$ \\
\hline Fat spreads & 10 & & 10 & & $5^{*}$ & & $10^{*}$ & & $5^{*}$ & & $5^{*}$ & & $5^{\star}$ \\
\hline \multicolumn{14}{|l|}{ Foods HFSS } \\
\hline Biscuits & 17 & $5-28$ & & & $10^{*}$ & & & & & & $10^{*}$ & & $10^{*}$ \\
\hline Ice cream & 53 & $40-69$ & $\begin{array}{c}50 \\
\text { (2 scoops) }\end{array}$ & & & & $\begin{array}{c}60^{\star} \\
\text { (1 scoop) }\end{array}$ & & & & & & \\
\hline Crisps/savoury snacks & 25 & $8-50$ & 30 & & & & $20^{*}$ & & & & & & \\
\hline Chocolate & 25 & $16-40$ & 25 & & & & $9^{\star}$ & & & & & & \\
\hline
\end{tabular}

NGO, non-governmental organisation; HCP, health-care professional; CIAA, Confederation of the Food and Drink Industries of the EU; WCRF, World Cancer Research Fund; BHF, British Heart Foundation; BDA, British Dietetic Association; DOM UK: Dietitians in Obesity Management UK; HFSS, high in fat, sugar or salt.

${ }^{*}$ Converted from a household measure or number of items into a weight.

tStarchy carbohydrate food portion size given in scheme was doubled to give true portion size. 
grapes, and medium fruit. The differences that existed in the industry portion sizes may indicate that the NHS 5-a-day message may not always be followed when communicating portion sizes through the products and packaging produced. This is of concern as consumers may believe they are consuming one portion of some fruits or vegetables produced by industry, but in fact they may be under- or overconsuming these foods. Overconsumption of fruit juice is of particular concern due to the high sugar content and lack of fibre highlighted by the 5-a-day message limiting a portion to $150 \mathrm{ml}$, yet the retailer suggested a $250 \mathrm{ml}$ portion.

In comparison with the NGO schemes, the BDA and DOM UK portion sizes were largely consistent for cooked vegetables, medium fruit and dried fruit, but not for berries and grapes ( $72 \mathrm{~g}$ for NGO $v .103 \mathrm{~g}$ for BDA and $65 \mathrm{~g}$ for DOM UK), small fruit (110 g for NGO $v .138 \mathrm{~g}$ for $\mathrm{BDA}$ and $153 \mathrm{~g}$ for DOM UK) and large fruit (125 $\mathrm{g}$ for NGO v. $100 \mathrm{~g}$ for BDA and $40 \mathrm{~g}$ for DOM UK). These differences were likely to have occurred as these schemes gave the portion sizes of these food categories with specific examples, whereas the others (WCRF, Diabetes UK and BHF) gave general portion sizes and thus on conversion into weights the average of several examples was given. Moreover, BDA and DOM UK stated that a portion of small fruits was two to three pieces, whereas it was two pieces for the NGO schemes; the reason for this difference is unclear.

\section{Breakfast cereals}

Breakfast cereal portion sizes were very similar between the two industry schemes, varying by a maximum of $5 \mathrm{~g}$ within each type of cereal. Across the NGO and HCP schemes, portion sizes continued to be consistent for flaky breakfast cereals, but they were slightly larger than industry (30 to $35 \mathrm{~g}$ for industry and 36 to $42 \mathrm{~g}$ for NGO and HCP), and for puffed rice breakfast cereals they were slightly smaller ( 30 to $35 \mathrm{~g}$ for industry and 24 to $28 \mathrm{~g}$ for NGO and HCP). Biscuit-type breakfast cereals portion sizes were also similar between industry and the BHF. However, for muesli, NGO and HCP portion sizes (60 to $105 \mathrm{~g}$ ) were between $20 \%$ and, exceptionally, $133 \%$ larger than industry portions ( 45 to $50 \mathrm{~g}$ ). Moreover, for crunchy cereals, NGO and HCP portions were between 20 and $56 \%$ larger than industry portions. The discrepancies in muesli and crunchy cereal portion sizes could be due to the NGO and HCP schemes providing a general portion size in household measures for all cereal types as this approach does not account for the varying densities of breakfast cereals. The BHF muesli portion size was closer to the industry portion size as the BHF gave separate portion sizes for muesli and flaky or puffed rice breakfast cereals. It could also be argued that the schemes did not intend the serving sizes of cereals to be doubled; indeed, they intend the serving size to be used in conjunction with the recommended frequency of daily servings. However, this would mean although denser breakfast cereals would have smaller portions more in line with industry portions, the portions of flaky and puffed rice cereals would be considerably smaller than the on-pack and industry regulatory body suggestions for a portion.

\section{Bread, rice, pasta and potatoes}

There was considerable disagreement across schemes with regard to the portion sizes of bread, rice, pasta and potatoes. The WCRF portion sizes stand out as they were often larger than those in the other general advice schemes (i.e. for bread and potatoes) and were consistently larger than those in the weight-loss advice schemes. This may reflect the amount that would need to be consumed to meet energy requirements if 'discretionary' items such as biscuits, confectionery and sugar-sweetened beverages were limited in the diet (and portion sizes for many of those and similar foods are not provided by WCRF).

Although the retailer portion sizes were highly variable for bread, the median portion size was 39 to $45 \%$ smaller compared with the NGO and HCP schemes. The incongruence with industry, where the on-pack suggested serving was likely to be one slice of bread (as on-pack nutrition information is often given per unit of food where applicable), was due to the doubling of the NGO and HCP servings of bread to give one 'portion' of two slices. However, this more realistically reflects common consumption of bread at one eating occasion, e.g. two slices of bread to make a sandwich (the mean weight eaten of bread from the National Diet and Nutrition Survey in adults conducted in year 2000/2001 was $73 \cdot 3 \mathrm{~g}$, which would be approximately two slices). The inconsistency between our conversion of $36 \mathrm{~g}$, the WCRF suggesting that one slice was $40 \mathrm{~g}$ and the industry median weight of a slice of bread being $44 \mathrm{~g}$ may indicate the standard conversions (based on data from 2002 ${ }^{(17)}$ ) are outdated and that currently an average commercially available slice of bread is larger than is presumed.

A portion size for rice of $160 \mathrm{~g}$ was consistent across most of the weight loss advice schemes. However, WCRF stands out with a portion size of $200 \mathrm{~g}$, although this is similar to the retailer median rice portion of $208 \mathrm{~g}$. The retailer and WCRF portion sizes for pasta were also quite similar. However, the CIAA portion size range (180-220 g) encompasses the WCRF portion but not the retailer median portion size or the weight-loss advice portion sizes (148g for Diabetes UK and DOM UK and $222 \mathrm{~g}$ for BHF and BDA). The consistency across rice portion sizes in general advice and weight-loss advice schemes, separately, is in contrast to the inconsistency across the pasta portion sizes.

For potatoes, portion sizes were consistent (160 g) among the weight-loss advice schemes, but were larger than the median potato portion size in the retailer scheme and smaller than the WCRF potato portion size $(240 \mathrm{~g})$. The variability in the portion sizes between schemes, and within the retailer scheme, for rice, pasta and potatoes, suggests that appropriate portion sizes still need to be defined. 


\section{Meat and fish}

The portion sizes for meat and poultry were similar across NGO and HCP schemes except for DOM UK which was $34 \%$ smaller on average. CIAA considers a portion to be approximately 33\% larger than the majority of the other schemes. The retailer median portion sizes for meat and poultry were variable; compared with the NGO and HCP schemes the median meat portion size was much smaller at $47 \mathrm{~g}$, while for poultry it was slightly larger at $100 \mathrm{~g}$. The Department of Health recommends that a portion of red meat should be $70 \mathrm{~g}$ (once daily) due to the increased risk of bowel cancer in those who consume more than $90 \mathrm{~g}$ of red or processed meat daily ${ }^{(19)}$. This indicates that schemes need to reduce their suggested meat portions to be in line with this recent government recommendation. It is notable that the CIAA gave a recommendation of $100 \mathrm{~g}$ for meat as a meal component, which was used in the present study, but an additional recommendation of $25 \mathrm{~g}$ for meat as a sandwich filler was given in the scheme ${ }^{(14)}$. Therefore, although the CIAA portion of meat as a meal component is larger than for other schemes, this scheme recognises the need to supply different portion size suggestions for different purposes. The inclusion of suggested portion sizes of meat for purposes such as for sandwiches (e.g. sliced ham) most likely explains why we found that the retailer median on-pack suggested portion size for meat to be considerably smaller than the others. However, the retailer portion size range extended to $200 \mathrm{~g}$ which is more than double the NGO and HCP recommendations. The suggested portion size for oily fish was variable across the schemes (75 to $140 \mathrm{~g}$ ), but was more consistent for white fish (124 to $150 \mathrm{~g}$ ).

\section{Legumes, nuts and seeds}

Across the schemes, the portion size of pulses was reasonably consistent, varying between approximately $120 \mathrm{~g}$ (retailer, BHF and BDA) and $150 \mathrm{~g}$ (WCRF and DOM UK). However, Diabetes UK gave a much smaller portion of $53 \mathrm{~g}$. This may be related to the conversion from household measures to absolute weights, which may have resulted in a smaller than intended portion. There was agreement among the NGO schemes that a portion of nuts was $50 \mathrm{~g}$ but industry schemes and DOM UK gave portion sizes 40 to $50 \%$ and $20 \%$ smaller, respectively, than the NGO. For seeds, the retailer and WCRF were in agreement on the portion size ( 24 to $25 \mathrm{~g}$ ); however, DOM UK gave a portion $60 \%$ larger.

\section{Dairy}

The suggested portion size for milk was very consistent across schemes (197-200 g), except for the larger portion suggested by WCRF (259g). Yoghurt or fromage frais portion sizes varied between schemes (100 to $162 \mathrm{~g}$ ), suggesting that the appropriate portion size for this food is still equivocal. The cheese portion sizes were congruent (30g), except for DOM UK which gave a $17 \%$ smaller portion.

\section{Oils and fats}

The NGO and HCP schemes, except for Diabetes UK, suggested portion sizes of oils and fats that were considerably smaller ( $3 \mathrm{~g}$ for oil and $5 \mathrm{~g}$ for butter and fat spreads) than industry portions ( $15 \mathrm{~g}$ for oil ( $400 \%$ larger) and $10 \mathrm{~g}$ for butter and fat spreads (100\% larger)). The smaller NGO and HCP suggestions for fats and oils portion sizes indicate that it may be important for industry to readdress not only the on-pack labelling and regulatory guidance for this group of foods, but also the widely available individual $10 \mathrm{~g}$ portions of butter and fat spreads. However, it is surprising that, despite being aimed at weight loss, the Diabetes UK suggested portions of these foods were twice as large compared with the other NGO and HCP schemes $(6 \mathrm{~g}$ for oil and $10 \mathrm{~g}$ for butter and fat spreads).

\section{Foods bigh in fat, sugar or salt}

When considering the foods high in fat, sugar or salt (HFSS), it is noteworthy that the biscuits, crisps and chocolate portion sizes commonly available to consumers, and suggested by the industry regulatory body, appeared to be larger than the suggested portion sizes from NGO and HCP (17 g $v .10 \mathrm{~g}$ for biscuits; 25 to $30 \mathrm{~g} v$. $20 \mathrm{~g}$ for crisps and snacks; $25 \mathrm{~g} v .9 \mathrm{~g}$ for chocolate).

For ice cream initially it appeared that suggested portion sizes from industry and Diabetes UK were in agreement. However, there is a discrepancy between the conversions of one scoop, as the suggested number of scoops was two for the CIAA and one for Diabetes UK. The conversion used in DINO is $60 \mathrm{~g}^{(17)}$, whereas the conversion used by the CIAA is $25 \mathrm{~g}^{(11)}$. Therefore one of the conversions could be incorrect which could perhaps be due to variations in the density of ice creams. However, as consumers prefer to follow guidelines based on household measures ${ }^{(5)}$, the inconsistency in the number of recommended scoops is of concern.

Several schemes (WCRF, BHF, BDA and DOM UK) did not give recommendations for three of the foods HFSS. This could suggest that either the recommendation was not to consume any of these foods, or that the schemes were unable to suggest a portion size as a general recommendation. However, the most likely explanation is that these schemes consider these foods as optional snacks that are picked by the individual based on the desired total daily energy intake. Indeed, some of these schemes (BHF, BDA and DOM UK) listed different portion sizes of foods HFSS and the corresponding energy content ${ }^{(20-22)}$.

\section{Discussion}

As one would expect, within the two industry schemes designed to give general advice, portion sizes were fairly consistent. Likewise, there was often consistency in schemes intended for weight-loss advice. However, the 
WCRF scheme, intended for general advice, was more consistent with the weight-loss schemes than the general advice schemes. Inconsistency across portion size messages between schemes intended for the same purpose (such as general advice or weight-loss advice) could exacerbate consumer concerns $^{(6)}$ about the credibility of portion size information. Similarities and differences between portion sizes could be explained by the differences in the rationale used to develop each scheme (Table 1).

The portion sizes suggested by DOM UK were often incongruent with the other NGO and HCP schemes intended for weight loss. They suggested smaller portions of cheese, meat, fish and nuts, but slightly larger portions of small fruit and pulses, shifting the overall balance of the diet away from protein and fat and towards carbohydrate-rich foods, perhaps to encourage closer adherence to dietary reference values for macronutrients. However it is not clear if these recommendations are appropriate for weight loss.

It is noteworthy that the CIAA (an industry regulatory body) did not specify portion sizes for many of the core food groups (except meat and poultry) focusing instead on 'processed' foods. However, often individual groups will have portion sizes for these food groups, e.g. The Dairy Council $^{(23)}$ or the NHS 5-a-day scheme ${ }^{(24)}$. This may explain the consistency between portion sizes suggested by the retailer and the NGO and HCP schemes for medium fruit, cooked vegetables, dried fruit, milk and cheese. However, for some foods, even if the industry median portion was consistent with suggested portion sizes from other schemes, often the range of suggested portion sizes was highly variable (e.g. pulses). This could lead to consumer confusion and subsequent distrust in on-pack portion size messages.

It is notable that sugar-sweetened beverages are not included in this comparison. The CIAA was the only scheme to give a suggested portion size for non-alcoholic beverages $(250 \mathrm{ml}$ is one portion in a multi-portion pack). However, given the association between sugarsweetened beverages and the risk of weight gain ${ }^{(25)}$, portion size advice for these products would be particularly helpful.

The sometimes marked difference between industry portion sizes and the recommendations of the NGO or HCP schemes, particularly for foods HFSS, suggests that the recommended portion sizes are not always the size of the commercially available pre-packaged units. This highlights the importance in considering the portion sizes of the products available to consumers when communicating recommendations for portion sizes.

\section{Limitations}

The current study has several limitations. First, not all foods were examined and there may be some foods such as other foods HFSS that would be important to consider due to their contribution to energy intake and subsequent importance in weight maintenance and weight loss. Second, only UK schemes have been considered, but the UK public may be exposed to some other overseas schemes (e.g. the US Department of Agriculture's My Pyramid scheme ${ }^{(26)}$ ) which may increase variability even further. Third, only schemes providing suggested portion sizes for adults were included in the current study. However, furthering this research to examine the consistency of portion sizes suggested in schemes intended for children would be warranted. Fourth, only one retailer was used as a representative of a business portion size scheme. Further retailer schemes would add more information on portion sizes commercially available and communicated by businesses. Finally, comparisons were made after objective conversions of household measures into weights. As there was not always sufficient information given in the schemes, the conversions may not always be consistent with the schemes' intentions. Moreover, conversion into weights is not necessarily how a member of the public would interpret the scheme and there is likely to be considerable inter-individual variability in portions served even when apparently adhering to the guidelines. Concluding whether the communication of portion sizes of some foods is consistent is difficult as the schemes sometimes gave insufficient information or DINO lacked exact conversions and the closest available conversion had to be used (i.e. only a heaped tablespoon conversion was available for nuts). Moreover, as previously found in the IGD report ${ }^{(1)}$, the examination of the schemes in the present study highlighted the inconsistency and lack of clarity in the use of the terms 'portion size' and 'serving size'. In addition, schemes often described portion sizes along with frequencies of consumption, which, as this caused a lack of ease of interpretation in the present study, could also lead to errors in interpretation by members of the public.

\section{Conclusions}

The present comparison has revealed the substantial discrepancies between recommended portion sizes in different schemes and that greater consistency in the portion sizes communicated to the public is needed. Advice to consumers could be improved if schemes were clearer as to the exact suggested portion size intended (e.g. a heaped or level tablespoon). These measures could help to improve the credibility of the portion size advice. Moreover, distinguishing between schemes intended for weight loss and those giving general advice would also help to address some of the public's confusion around portion size recommendations ${ }^{(6)}$. The variability within a food type in the industry on-pack suggested portion sizes may contribute to distrust in industry portion size advice and the inability to form a concept of an appropriate portion. As current NGO and HCP schemes 
may only be accessed by health-conscious members of the public or those wishing to lose weight, industry schemes may have a greater impact as exposure is across all consumers. There may be benefits of mass communication of an independent and authoritative scheme on suggested portion sizes for all foods, with distinct recommendations for general advice and for weight-loss advice.

\section{Acknowledgements}

Source of funding: This study was conducted by MRC Human Nutrition Research (U105960389), funded by the UK Medical Research Council. Conflicts of interest: S.A.J. is the independent chair of the Public Health Responsibility Deal Food Network for England. Authors' contributions: H.B.L. and S.A.J. designed the study. H.B.L. carried out the data acquisition, analysis and interpretation, and drafted the manuscript. S.A.J. and A.L.A. contributed to the data interpretation and critical revision of the manuscript. Acknowledgements: The authors thank Nida Ziauddeen for providing the conversions used in this study. They also thank the UK supermarket that provided data for the study.

\section{References}

1. Institute of Grocery Distribution (2008) Portion Size: A Review of Existing Approaches. Watford: Institute of Grocery Distribution.

2. Butland B, Jebb S, Kopelman P et al. (2007) Foresight Tackling Obesities: Future Choices - Project Report, 2nd ed. London: Government Office for Science.

3. Kelly MT, Wallace JMW, Robson PJ et al. (2009) Increased portion size leads to a sustained increase in energy intake over $4 \mathrm{~d}$ in normal-weight and overweight men and women. Br J Nutr 102, 470-477.

4. Schwartz J \& Byrd-Bredbenner C (2006) Portion distortion: typical portion sizes selected by young adults. J Am Diet Assoc 106, 1412-1418.

5. Institute of Grocery Distribution (2009) Portion Size: Understanding the Consumer Perspective. Working Group Report. Watford: Institute of Grocery Distribution.

6. Anderson AS, Barton K, Craigie A et al. (2008) Exploration of Adult Food Portion Size Tools. Edinburgh: NHS Scotland.

7. The Confederation of the Food and Drink Industries of the EU (2010) Portion Sizes For Purposes of Nutrition Labelling CIAA Proposed Approach. http://gda.ciaa.eu/asp2/portion sizes_nutrition_labelling.asp?doc_id=235 (accessed November 2010).

8. Association Internationale de l'Industrie des Bouillons et Potages, Federation des Associations de l'Industrie des Bouillons et Potages de la CEE (2009) Portion Sizes for Soups in Europe. http://gda.ciaa.eu/custom_documents/ documents/operators/portion_sizes_for_soups.pdf (accessed November 2010).

9. Association of the Chocolate Biscuits and Confectionery Industries of the EU (2009) Rationale of CAOBISCO Guideline for Reference Quantity for the Purpose of GDA Labelling. http://gda.ciaa.eu/custom_documents/documents/ operators/caobisco_rationale.pdf (accessed November 2010).
10. European Breakfast Cereal Association (2009) CEEREAL Portion Sizes. http://gda.ciaa.eu/custom_documents/ documents/operators/CEEREAL\%20Portion\%20Size\%20 Rationale.pdf (accessed November 2010).

11. European Ice Cream Association (2010) Rationale with regard to the Portion Size for purposes of Nutrition Labelling. http://gda.ciaa.eu/custom_documents/documents/ operators/Euroglaces_portion_sizes.pdf (accessed November 2010).

12. European Snacks Association (2010) Portion Rationale. http://gda.ciaa.eu/custom_documents/documents/operators/ ESA\%20Portion\%20Rationale\%20Sheet\%20July\%202010.pdf (accessed November 2010).

13. International Margarine Association of the Countries of Europe (2010) Margarine Portion IMACE Rationale. http:// gda.ciaa.eu/custom_documents/documents/operators/imace_ margarine_portion.pdf (accessed November 2010).

14. Meat Processing Industry in the EU (2010) CLITRAVI view on portion sizes. http://gda.ciaa.eu/custom_documents/ documents/operators/CLITRAVI_view_on_portion_sizes.pdf (accessed November 2010).

15. Union of European Beverages Associations (2009) UNESDA rationale for the generally-recommended portion in relation to GDA nutrition labelling of non-alcoholic beverages in the EU. http://gda.ciaa.eu/custom_documents/ documents/operators/UNESDA\%20GDAs\%20portion\%20 rationale.pdf (accessed November 2010).

16. Union of Organizations of Manufacturers of Pasta Products of the EU (2010) UNAFPA Portion Sizes. http://gda.ciaa.eu/ custom_documents/documents/operators/UNAFPA_portionsrationale.pdf (accessed November 2010).

17. Food Standards Agency (2002) Food Portion Sizes. London: The Stationary Office.

18. Food Standards Agency (2002) McCance and Widdowson's The Composition of Foods. Cambridge: Royal Society of Chemistry.

19. Department of Health (2011) Red meat link to bowel cancer. http://www.dh.gov.uk/en/MediaCentre/Pressreleases/DH_124670 (accessed April 2011).

20. British Heart Foundation (2009) So you want to lose weight...for good. A guide to losing weight for men and women. http://www.bhf.org.uk/publications/view-publication. aspx?ps $=1000807$ (accessed April 2011).

21. British Dietetic Association (2006) Eating Well: your weight wise plan. http://www.bdaweightwise.com/eating/eating_ plan.html (accessed November 2010).

22. Dieticians in Obesity Management UK (2005) Controlling your portions. http://www.gcu.ac.uk/sndri/all_health_ resources.html\#DOMUK (accessed March 2011).

23. The Dairy Council (2007) Dairy and Obesity. http://www. milk.co.uk/page.aspx?intPageID $=159$ (accessed November 2010).

24. National Health Service (2009) 5 A DAY portion sizes. http://www.nhs.uk/Livewell/5ADAY/Pages/Portionsizes.aspx (accessed November 2010).

25. Malik VS, Schulze MB \& Hu FB (2006) Intake of sugarsweetened beverages and weight gain: a systematic review. Am J Clin Nutr 84, 274-288.

26. US Department of Agriculture (date not known) MyPyramid. gov: Steps to a healthier you. http://www.mypyramid.gov/ (accessed April 2011)

27. World Cancer Research Fund (2009) What Does a Healthy Portion Look Like? http://www.wcrf-uk.org/PDFs/Healthy PortionPad.pdf (accessed November 2010).

28. Diabetes UK (2008) Calorie controlled plan. http:// www.diabetes.org.uk/Guide-to-diabetes/Healthy_lifestyle/ Managing_your_weight/Calorie_controlled_plan/ (accessed November 2010). 